\title{
Terminal Sliding Mode Control of MIMO Linear Time Delay Systems
}

\section{Zhongqiang WU}

Institute of Electrical Engineering, Yanshan University, Hebei, 066004, China

email: mewzq@163.com

Keywords: Terminal sliding mode, time delay, variable structure control, finite time convergence.

\begin{abstract}
A new terminal sliding mode control of MIMO linear time delay system is proposed in this paper. By inserting a time delay term in sliding mode, a new terminal sliding mode is developed for MIMO linear time delay systems. A terminal sliding mode controller is designed to drive the system state variables to reach the terminal sliding mode with a desired finite time and stay there forever. By suitably designing the parameter matrices of the sliding mode, the system state variables reach the system origin in finite time.
\end{abstract}

\section{Introduction}

Recently, a new technique called terminal sliding mode control has been developed in [1]-[5]. It offers some superior properties such as finite time convergence of the system dynamics in the terminal sliding mode and less steady state errors. In [1]-[3], the first-order terminal sliding mode control technique is developed and is applied into second-order nonlinear system and nth-order nonlinear rigid robotic manipulator. In [4], the terminal sliding mode technique for high-order single-input and single-output linear system is studied. In [5], the terminal sliding mode control of MIMO systems is investigated and a new terminal sliding mode structure is got.

The systems studied in the papers presented above haven't including time delay. However time delay systems can be found in various industrial systems, such as electrical networks, rolling mill, chemical processes, and hydraulic systems. In such a situation, designing a controller without taking into account the delay may lead to unstable or poor performance. The study of time delay systems has received considerable attention over the past years. The variable structure control of the time delay systems has been well studied in many literatures [6]-[8]. The results about independent of the delay and delay dependent have been got. However the terminal sliding mode control for linear time delay system is rare. It needs to be investigated.

This article is organized as follow. Section 2 describes the proposed new terminal sliding mode control scheme for MIMO linear time delay systems. Section 3 A simulation example is studied and a comparison is made. Section 4 gives conclusion.

\section{Terminal sliding mode control for MIMO linear time delay systems}

Consider the following MIMO linear time delay systems:

$$
\begin{aligned}
& \dot{X}_{1}(t)=A_{11} X_{1}(t)+A_{12} X_{2}(t)+A_{d 11} X_{1}(t-h) \\
& \dot{X}_{2}(t)=A_{21} X_{1}(t)+A_{22} X_{2}(t)+A_{d 21} X_{1}(t-h)+A_{d 22} X_{2}(t-h)+B_{2} U(t)
\end{aligned}
$$

where $X_{1}(t) \in R^{n-m}$ and $X_{2}(t) \in R^{m}$ are system states, $A_{11} \in R^{(n-m) \times(n-m)}, A_{12} \in R^{(n-m) \times m}$, $A_{d 11} \in R^{(n-m) \times(n-m)}, A_{21} \in R^{m \times(n-m)}, A_{22} \in R^{m \times m}, A_{d 21} \in R^{m \times(n-m)}, A_{d 22} \in R^{m \times m}$ and $B_{2} \in R^{m \times m}$ are the system matrices. $B_{2}$ is non-singular, and $n-m \leq m . h=$ const $>0$ is pure time delay.

A new terminal sliding variable vector for the above MIMO linear time delay system is defined as follows:

$$
S=C_{1} X_{1}(t)+X_{2}(t)+C_{2} X_{1}(t)^{\frac{q}{p}}+C_{3} X_{1}(t-h)
$$

where $C_{1}, \quad C_{2}, \quad C_{3} \in R^{m \times(n-m)}$ are the parameter matrices of the terminal sliding mode. The odd 
integer $\mathrm{p}$ and $\mathrm{q}$ satisfy the following condition.

$$
p>q \text {, and } 2 q>p
$$

$X_{1}(t)^{\frac{q}{p}}$ is a vector defined as

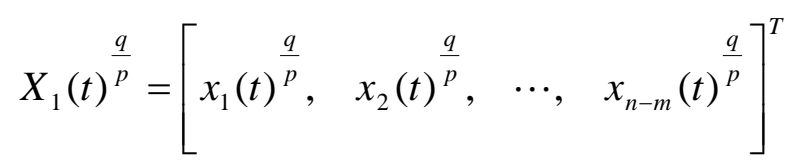

The terminal sliding mode is then defined as $S=0$ or

$$
C_{1} X_{1}(t)+X_{2}(t)+C_{2} X_{1}(t)^{\frac{q}{p}}+C_{3} X_{1}(t-h)=0
$$

Remark 1: It can be seen from (6) that the structure of the terminal sliding mode is different from the one in [5] due to the fact that the term $C_{3} X_{1}(t-h)$ is inserted in the sliding mode.

To obtain the sufficient condition of the existence of the terminal sliding mode (6) for the MIMO linear tine delay system in (1) and (2), we have the following theorem.

Theorem 1:

Consider the MIMO linear time delay system in (1) and (2). If the control input is designed such as (7),

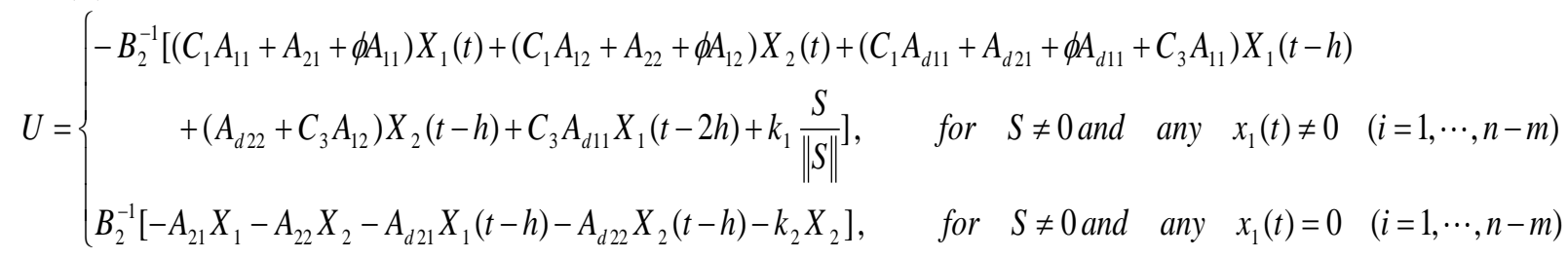

where $\phi=\frac{q}{p} C_{2} \operatorname{diag}\left(x_{i}(t)^{\frac{(q-p)}{p}}\right)$, and

$$
\operatorname{diag}\left(x_{i}(t)^{\frac{(q-p)}{p}}\right)=\left[\begin{array}{lll}
x_{1}(t)^{\frac{(q-p)}{p}} & & \\
& \ddots & \\
& & x_{n-m}(t)^{\frac{(q-p)}{p}}
\end{array}\right]
$$

$k_{1}$ and $k_{2}$ are positive numbers, then the system state variables will reach the terminal sliding mode $S=0$ in finite time.

Proof: Defining a Lyapunov function

$$
V=\frac{1}{2} S^{T} S
$$

and differentiating $V$ with respect to time, we have $\dot{V}=S^{T} \dot{S}$

$$
\begin{aligned}
= & S^{T}\left[C_{1} \dot{X}_{1}(t)+\dot{X}_{2}(t)+\frac{q}{p} C_{2} \operatorname{diag}\left(x_{i}(t)^{\frac{(q-p)}{p}}\right) \dot{X}_{1}(t)+C_{3} \dot{X}_{1}(t-h)\right] \\
= & S^{T}\left[\left(C_{1} A_{11}+A_{21}+\varphi A_{11}\right) X_{1}(t)+\left(C_{1} A_{12}+A_{22}+\varphi A_{12}\right) X_{2}(t)+\left(C_{1} A_{d 11}+A_{d 21}+\varphi A_{d 11}+C_{3} A_{11}\right) X_{1}(t-h)\right. \\
& \left.\quad+\left(A_{d 22}+C_{3} A_{12}\right) X_{2}(t-h)+C_{3} A_{d 11} X_{1}(t-2 h)+B_{2} U\right],
\end{aligned}
$$

Using (7) in (10), we get

$$
\dot{V}=-k_{1}\|S\|<0 \quad \text { for } S \neq 0 \quad \text { and } \quad x_{i}(t) \neq 0 \quad(i=1, \cdots, n-m)
$$

Expression (11) is the sufficient condition for the terminal sliding variable vector $S$ to converge to zero in finite time according to Lyapunov stability theory [1]-[5].

Remark 2: It can be seen from the above theorem that the convergence of the sliding variable vector $S$ is obtained under the condition that $S \neq 0$ and $x_{i}(t) \neq 0 \quad(i=1, \cdots, n-m)$ because, at the 
point $S \neq 0$ and $\quad x_{i}(t)=0 \quad(i=1, \cdots, n-m), S^{T} S$ is unbounded. In order to make the system dymanics get away from these singular points, the second part of the control law in (7) is used, which is rewritten as

$$
U=B_{2}^{-1}\left[-A_{21} X_{1}-A_{22} X_{2}-A_{d 21} X_{1}(t-h)-A_{d 22} X_{2}(t-h)-k_{2} X_{2}\right] \text {. }
$$

Using (12) in (2), we have

$$
\dot{X}_{2}(t)=-k_{2} X_{2}(t)
$$

and the solution of the above differential equation has the following form:

$$
X_{2}(t)=K e^{-k_{2} t}
$$

where $K=\left[k_{1}, \cdots, k_{m}\right]^{T}$ is a constant vector.

Using (14) in (1), we have

$$
\dot{X}_{1}(t)=A_{11} X_{1}(t)+A_{12} K e^{-k_{2} t}+A_{d 11} X_{1}(t-h)
$$

It is easy to see that, the control signal $K e^{-k_{2} t}$ will drive the elements of $X_{1}(t)$ away from zero at the beginning, and after that the first part of the terminal sliding mode control law in (7) works again to drive the system dynamics into the terminal sliding mode.

The design of the parameter matrices of the terminal sliding mode in (6) and the convergence property of the MIMO linear tine delay system dynamics in the terminal sliding mode are states in the following theorem.

Theorem2: Consider the MIMO linear time delay system in (1) and (2) with the terminal sliding mode controller in (7). If the terminal sliding mode parameter matrices are designed such that

$$
\begin{aligned}
& A_{11}-A_{12} C_{1}=0 \\
& A_{d 11}-A_{12} C_{3}=0 \\
& A_{12} C_{2}=\operatorname{diag}\left(\beta_{i}\right)
\end{aligned}
$$

where $\beta_{i}>0, \quad i=1, \cdots, n-m$

Proof: According the terminal sliding mode (6), the state vector $X_{2}(t)$ can be expressed as

$$
X_{2}(t)=-C_{1} X_{1}(t)-C_{2} X_{1}(t)^{\frac{q}{p}}-C_{3} X_{1}(t-h)
$$

Using (19) in (1), we have

$$
\dot{X}_{1}(t)=\left(A_{11}-A_{12} C_{1}\right) X_{1}(t)-A_{12} C_{2} X_{1}(t)^{\frac{q}{p}}+\left(A_{d 11}-A_{12} C_{3}\right) X_{1}(t-h)
$$

Considering (16), (17) and (18), (20) becomes

$$
\dot{X}_{1}(t)=-\operatorname{diag}\left(\beta_{i}\right) X_{1}(t)^{\frac{q}{p}}
$$

or $\quad \dot{x}_{i}(t)=-\beta_{i} x_{i}(t)^{\frac{q}{p}} \quad i=1, \cdots, n-m$

The relaxation time $t_{i}$ for a solution of the system (22) is then given by

$$
t_{i}=-\beta_{i}^{-1} \int_{x_{i}(0)}^{0} \frac{d x_{i}(t)}{x_{i}(t)^{\frac{q}{p}}}=\frac{\left|x_{i}(0)\right|^{\frac{1-q}{p}}}{\beta_{i}(1-q / p)}
$$

then, $X_{1}(t)$ reaches the system origin in finite time. According to (19), therefore $X_{2}(t)$ identically reaches the system origin in finite time.

\section{A simulation example}

At first, the following MIMO linear system is considered [5]:

$$
\dot{x}_{1}=\left[\begin{array}{ll}
1 & 0
\end{array}\right]\left[\begin{array}{l}
x_{2} \\
x_{3}
\end{array}\right]
$$




$$
\left[\begin{array}{l}
\dot{x}_{2} \\
\dot{x}_{3}
\end{array}\right]=\left[\begin{array}{l}
0 \\
-1
\end{array}\right] x_{1}+\left[\begin{array}{cc}
0 & 1 \\
-2 & -3
\end{array}\right]\left[\begin{array}{l}
x_{2} \\
x_{3}
\end{array}\right]+\left[\begin{array}{ll}
0 & 1 \\
1 & 0
\end{array}\right]\left[\begin{array}{l}
u_{1} \\
u_{2}
\end{array}\right]
$$

In [5], the system is controlled with a good performance showing in Fig 1. The initialize states is $\left[\begin{array}{lll}0.15 & 0.09 & -0.15\end{array}\right]^{T}$

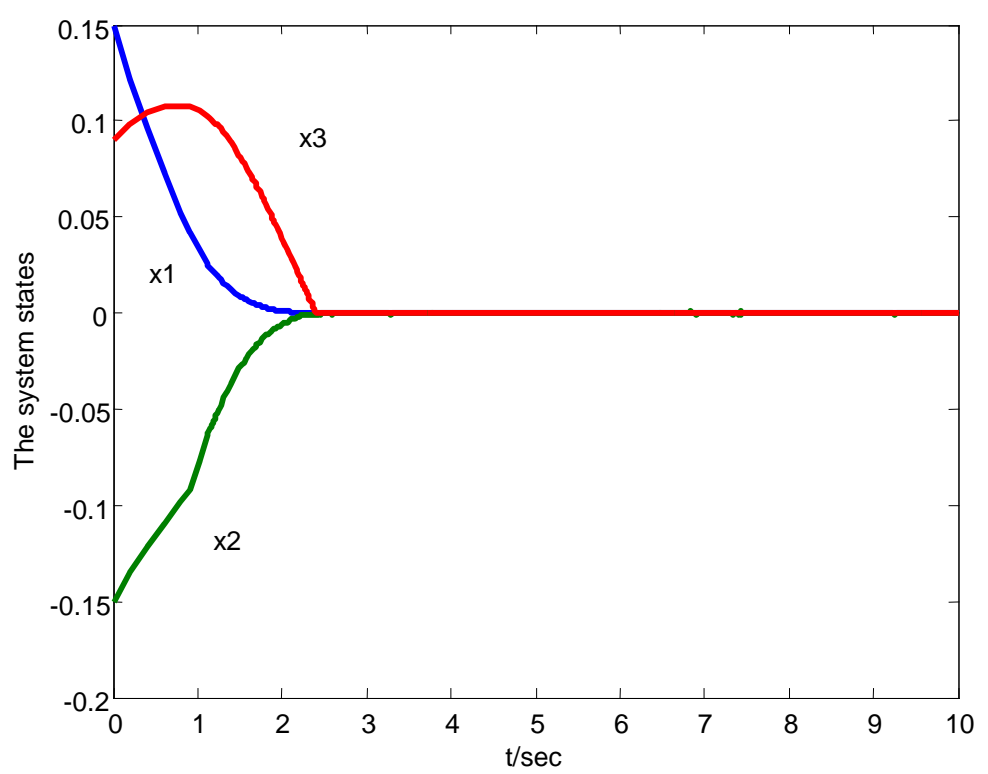

Fig 1. The system states in [5]

When we add the time delay, so the system can be given as follow:

$$
\begin{gathered}
\dot{x}_{1}=\left[\begin{array}{ll}
1 & 0
\end{array}\right]\left[\begin{array}{l}
x_{2} \\
x_{3}
\end{array}\right]+x_{1}(t-h) \\
{\left[\begin{array}{l}
\dot{x}_{2} \\
\dot{x}_{3}
\end{array}\right]=\left[\begin{array}{l}
0 \\
-1
\end{array}\right] x_{1}+\left[\begin{array}{cc}
0 & 1 \\
-2 & -3
\end{array}\right]\left[\begin{array}{l}
x_{2} \\
x_{3}
\end{array}\right]+\left[\begin{array}{l}
1 \\
1
\end{array}\right] x_{1}(t-h)+\left[\begin{array}{ll}
1 & 0 \\
0 & 1
\end{array}\right]\left[\begin{array}{l}
x_{2}(t-h) \\
x_{3}(t-h)
\end{array}\right]+\left[\begin{array}{ll}
0 & 1 \\
1 & 0
\end{array}\right]\left[\begin{array}{l}
u_{1} \\
u_{2}
\end{array}\right]}
\end{gathered}
$$

$h$ is pure time delay. Adopting the controller in [5], even if $h=10^{-10}$, the system can't be stabilized. The respond curve in 5 seconds is as in Fig 2.

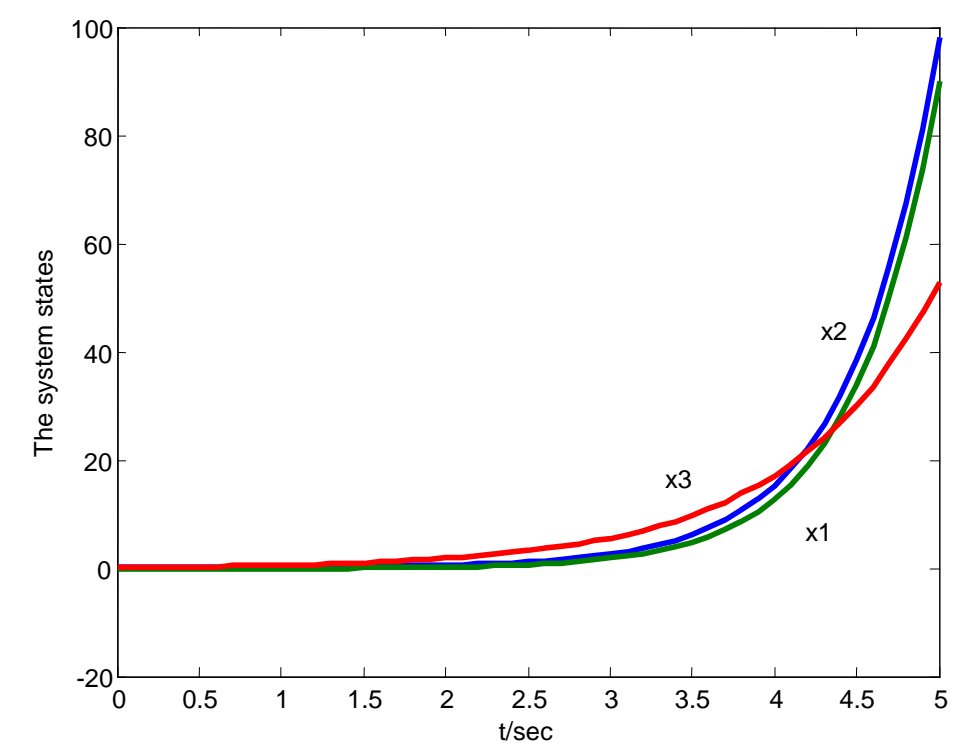

Fig 2. The time delay systems states with controller designed in [5]

The controller in this paper is adopted. where $A_{11}=0, A_{12}=\left[\begin{array}{ll}1 & 0\end{array}\right]^{T}, \quad A_{21}=\left[\begin{array}{ll}0 & -1\end{array}\right]^{T}$, 
$A_{22}=\left[\begin{array}{cc}0 & 1 \\ -2 & -3\end{array}\right], \quad B_{2}=\left[\begin{array}{ll}0 & 1 \\ 1 & 0\end{array}\right], \quad A_{d 11}=1, \quad A_{d 21}=\left[\begin{array}{ll}1 & 1\end{array}\right]^{T}, \quad A_{d 22}=\left[\begin{array}{ll}1 & 0 \\ 0 & 1\end{array}\right], \quad$ taking $\quad h=1$, $k_{1}=k_{2}=0.1$.

The terminal sliding variable vector is define as

$$
S=C_{1} x_{1}(t)+x_{2}(t)+C_{2} x_{1}(t)^{\frac{3}{4}}+C_{3} x_{1}(t-1)
$$

where $C_{1}=\left[\begin{array}{l}0 \\ 1\end{array}\right], C_{2}=\left[\begin{array}{l}1 \\ 0\end{array}\right], C_{3}=\left[\begin{array}{l}1 \\ 1\end{array}\right]$.

The controller is as follow:

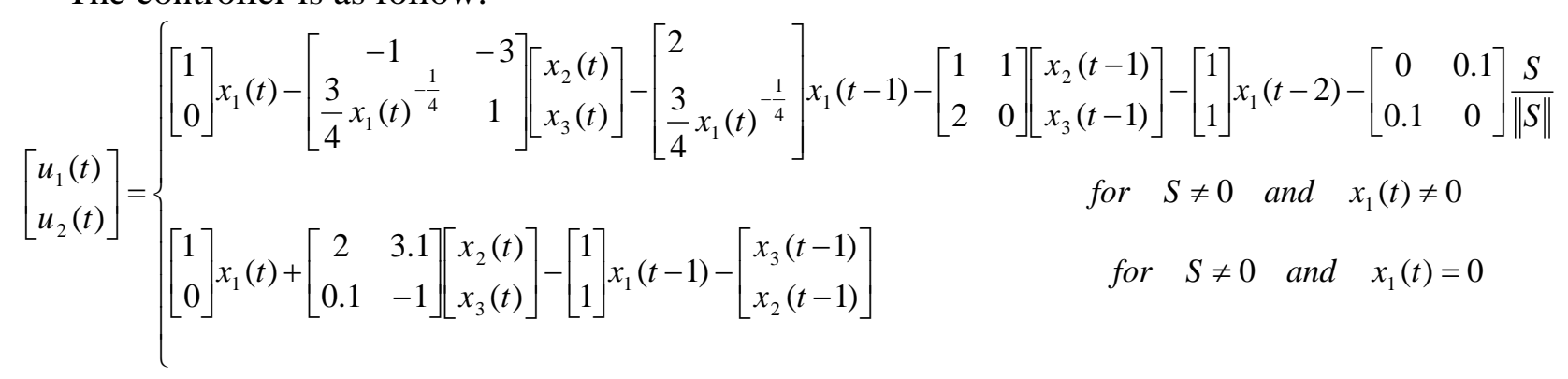

The simulation result is as in Fig 3. The system is stable and good performance is got.

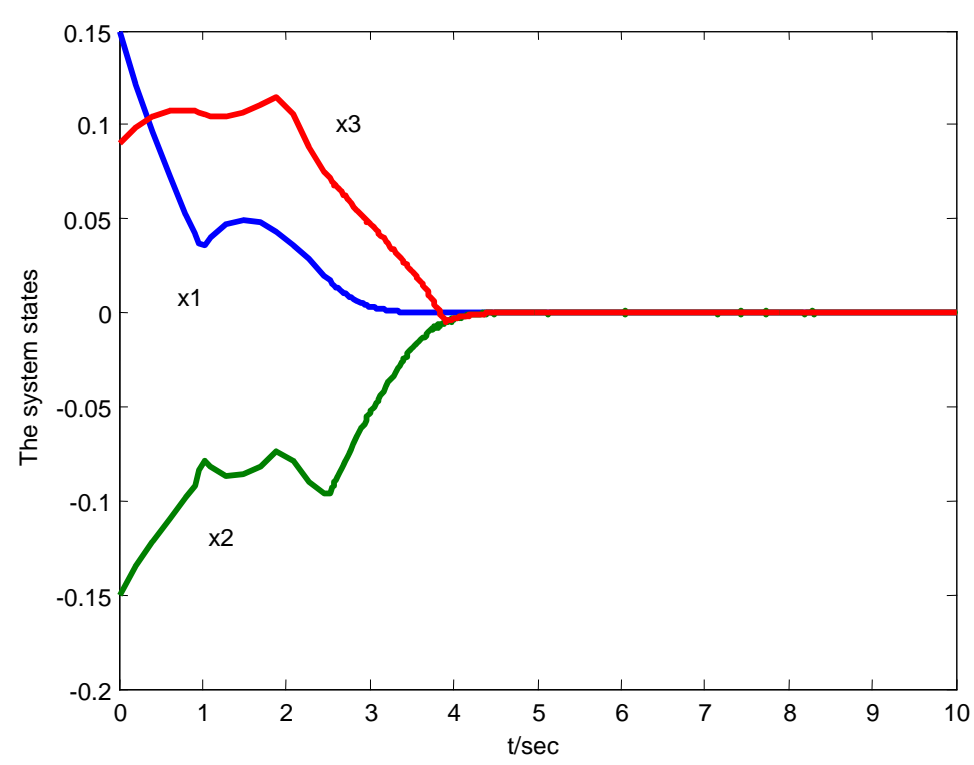

Fig 3. The time delay system states using the controller designed in this paper

\section{Conclusion}

In this paper, a new terminal sliding mode control scheme for MIMO linear time delay systems is got. The states variable of system can reach the terminal sliding mode in finite time. By suitably designing the parameter matrices of the terminal sliding mode, the states variable of system can reach the system origin in finite time. The simulation example shows the effect of the scheme in this paper. A comparison is made to show the scheme in paper [5] can't control the systems with time delay, even if the time delay is very small.

\section{Acknowledgement}

In this paper, the research was sponsored by the Nature Science Foundation of Hebei Province (Project No. F2016203006). 


\section{References}

[1] S. T. Venkataraman and S. Gulati, Control of nonlinear systems using terminal sliding modes, in Proc. Amer. Contr. Conf., 1992: 891-893

[2] Z. H. Man, A. P. Paplinski, and H. R. Wu, A robust MIMO terminal sliding mode control scheme for rigid robotic manipulators, IEEE Trans. Automat. Contr., 1994, 39(12): 2464-2469

[3] R. J. Stonier and X. H. Yu, eds, A robust neural controller for a class of second-order nonlinear system, Amsterdam, The Netherlands: IOS, 1994: 261-267

[4] X. H. Yu and M. Zhihong, Model reference adaptive control systems with terminal sliding modes, Int. J. Contr., 1966, 64(6): 1165-1176.

[5] Z. H. Man and X. H. Yu, Terminal sliding mode control of MIMO linear systems, IEEE Transaction on Circuit and Systems-I: Fundamental Theory and applications, 1997, 44(11):1065-1070.

[6] H. Choi, An LMI approach to sliding mode control design for a class of uncertain time delay systems. Proc. The European Control Conference ECC’99, Karlsruhe, Germany, 1999: 1025-1027,

[7] R. EL-Khazaly, Variable structure robust control of uncertain time delay systems. Automatica, 1998, 34(3): 327-332.

[8] F. Gouaisbaut, M. Damabrine. And J. P. Richard, Sliding mode control of linear time delay systems: a design via LMIs, IMA Journal of Mathematical Control and Information, 2002, 19(1 and 2): 83-94. 